\title{
Glucocorticoid management in rheumatoid arthritis: morning or night low dose?
}

\section{Sabrina Paolino, Maurizio Cutolo, Carmen Pizzorni}

Research Laboratories and Academic Division of Clinical Rheumatology Department of Internal Medicine, University of Genova, Italy

\begin{abstract}
Morning symptoms of rheumatoid arthritis (RA) are linked to circadian increase of night inflammation, supported by inadequate cortisol secretion in active disease. Therefore, exogenous glucocorticoid administration in RA is recommended by EULAR and ACR from the beginning of the diagnosis, since may partially act like a "replacement therapy". In addition, the prevention/treatment of the night up-regulation of the immune/inflammatory reaction has been shown more effective when exogenous glucocorticoid administration is managed with a night-time-release formulation.

Despite a considerably higher cost than conventional prednisone (immediate release), chronotherapy with night-time-release prednisone has been recognized a cost-effective option for RA patients not on glucocorticoids who are eligible for therapy with biologic disease-modifying antirheumatic drugs (DMARDs). Interestingly, since different cell populations involved in the inflammatory process are particularly activated during the night (i.e. monocytes, macrophages), other therapeutical approaches used in RA, such as conventional DMARDs and non-steroidal anti-inflammatory drugs (NSAIDs) should follow the same concepts of glucocorticoid chronotherapy. Therefore, bedtime methotrexate chronotherapy was found to better manage RA symptoms, and several available NSAIDs (i.e. indomethacin, aceclofenac, ketoprofen, flurbiprofen, lornoxicam) have been recently modified in their formulation, in order to obtain more focused night action.
\end{abstract}

Key words: rheumatoid arthritis, circadian rhythms, glucocorticoids, chronotherapy, disease-modifying antirheumatic drugs, non-steroidal anti-inflammatory drugs, rheumatic diseases.

\section{Introduction}

The crucial clinical signs and symptoms of rheumatoid arthritis (RA) vary within a day as well as between days, and in particular the morning joint stiffness that is present in almost all patients with active RA is considered one of the most characteristic diagnostic criteria of the disease [1].

On the other hands, other RA symptoms, that include joint pain and functional disability are commonly most severe in the early morning and restart following 24-h cycles [2]. The circadian rhythms of the disease are consequence of altered immune/inflammatory and neuroendocrine activities [2].

Since the night activation of the immune-inflammatory reaction is a common pathway, it is not surprising that also in other chronic inflammatory rheumatic dis- eases, like polymyalgia rheumatica (PMR) and ankylosing spondylitis, the symptoms of joint pain and stiffness typically are most evident during the early morning [3].

In synthesis, the morning symptoms, are linked to the circadian increase of proinflammatory mediators especially cytokines, as result of an increased night inflammation, at least in RA.

Cytokines, such as tumor necrosis factor $\alpha$ (TNF- $\alpha$ ) or interleukin 6 (IL-6) are highly effectively increased in patients with active RA in the very late night, whereas on the contrary are detectable at very low levels after 12 am [4].

It is now well assessed that circadian rhythms play an important role in RA symptomatology and both are linked to the pathophysiology of the disease $[5,6]$.

Among the triggers/enhancers for the increased cell release and serum concentrations of cytokines, are

Address for correspondence:

Maurizio Cutolo, Research Laboratories and Academic Division of Clinical Rheumatology, Department of Internal Medicine, University of Genova, IRCCS San Martino, Viale Benedetto XV, 6, 16132 Genova Italy, tel. +39 010353 7994, tel. +39 0103538885 ,

fax +39010 353 8885, mobile (+39) 3352336 21, e-mail: mcutolo@unige.it

Submitted: 16.07.2017; Accepted: 26.07.2017 


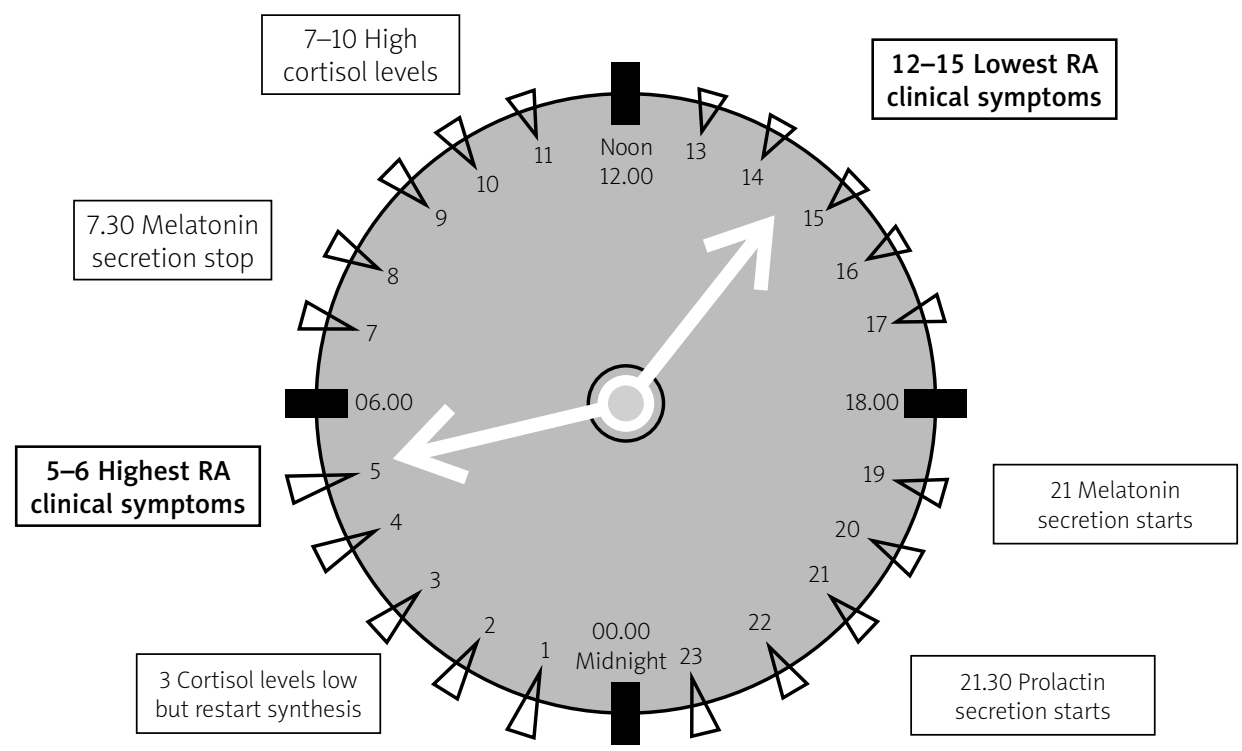

Fig. 1. The circadian sequence of nocturnal hormone secretion that induce activation (melatonin, prolactin) and/or down regulation (cortisol) of the immune inflammatory response, during the night, determine the clinical consequences that include morning symptoms such as joint stiffness and pain.

recognized the proinflammatory night hormones, such as melatonin (and prolactin), that follow theirself a 24hour daily cycle, as well as full availability of bioenergies during the night [7, 8] (Fig. 1).

In addition, the nocturnal capability of the neuroendocrine system to determ an intense inflammatory response with related clinical effects, seems also realized in acute inflammatory events such as the gout attack.

\section{The "clock" of circadian rhythms and rheumatoid arthritis}

In normal conditions and in all individuals, a circadian clock drives rhythms that are necessary to synchronise almost all the human functions according to the 24-hour environment [9].

Indeed, the most evident physiological functions under circadian control include the sleep-wake cycle, body temperature, blood pressure, heart rate, as well as endocrine regulation (i.e. adrenal and gonadal steroidogenesis) and in particulat the immune response.

Daily rhythms are controled by a central nervous system pacemaker, which is found in a hypothalamic region located above the optic chiasm and called the suprachiasmatic nucleus (SCN) [10] (Fig. 2).

Therefore, the SCN collects from the eyes the light inputs via the retinohypothalamic tract and the central pacemaker synchronises with other and additional periferal pacemakers found locally within organs, tissues and cells [10]. Interestingly, these "periferal clocks" are synchronised by the "central clock", however, at the same time, they are self-sustaining and can be entrained by external stimulators such as temperature changes.

Recently, a bi-directional interaction between the inflammatory reaction and the circadian clock has been shown. The disruption of the clock has a significant effect on the efficiency of the immune system, with impact on the pathogenesis of RA. On the contrary, the inflammatory reaction can directly alter cellular expression of clock genes [11].

Several possible disruptors of the clock have been identified and include for example the jet-lag, causing a de-synchrony between the internal clock and the environment, as well as the condition of night shift-workers.

In general, constant and frequent shifts in the daily schedule are strongly detrimental to health and have been implicated for an increased incidence of chronic diseases such as diabetes, irritable bowel syndrome, cardiovascular disease, metabolic syndromes and even cancer [12]. As matter of fact, a recent study showed a significant link between shift work and an increased risk of RA, especially in women [13].

The endocrine system mediates the diffusion of timing signals from the SCN throughout the body and to the immune system, having as circadian agents two night hormones, namely melatonin (prolactin) and glucocorticoids. Both hormones are now recognized crucial in regu- 


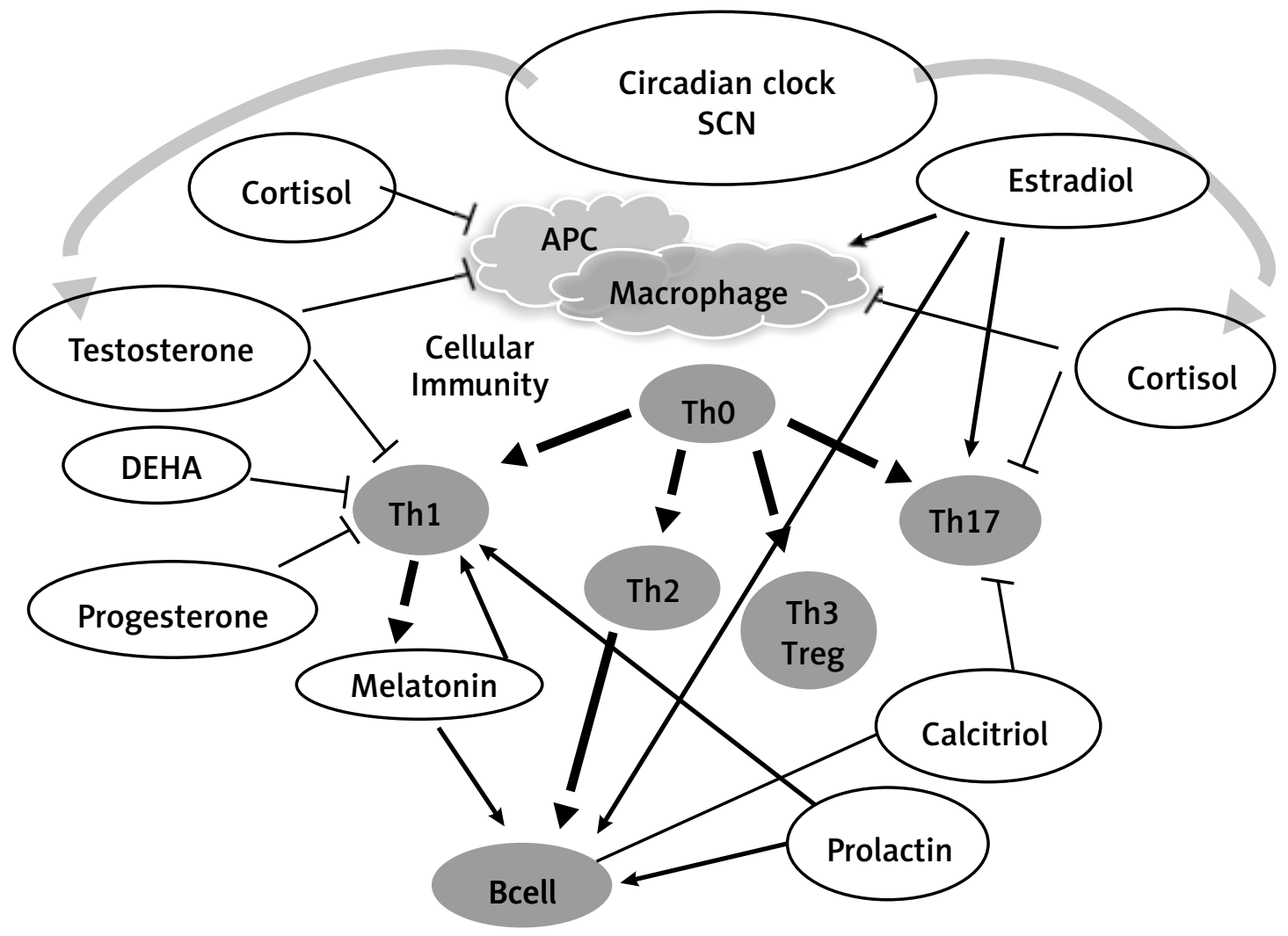

APC - Antigen Presenting Cell; Th - T helper cell; Treg - Regulatory T cell

Fig. 2. The daily neuroimmunoendocrine rhythms (gonadal, adrenal, pituitary hormones) are controlled by a central pacemaker, which is found in a hypothalamic region called the suprachiasmatic nucleus (SCN) of the central nervous system (CNS). Basically melatonin increases and cortisol reduces the Immune/inflammatory reaction following a circadian rhythm.

lation of the immune/inflammatory response, and therefore contribute to the pathogenesis of RA [14, 15] (Fig. 2).

\section{The endogenous glucocorticoid production in rheumatoid arthritis}

Generally, acute inflammatory events such as a bacterial infections, activate the response of the hypothalamus-pituitary-adrenal (HPA) axis leading to increased circulating $\mathrm{ACTH}$ and cortisol for a short period of time [16].

From the other side, the inflammation-associated down regulation of the HPA axis activity in chronic conditions such as in RA, depends on both to circulating cytokines that can damage the HPA axis functions on all levels (hypothalamus, pituitary gland and adrenal gland) and to a consequent and partial adrenal subclinical insufficiency [17] (Fig. 2).
The proinflammatory cytokines $\mathrm{IL}-1 \beta$ and TNF are the main factors, which interfere with several steps of steroidogenesis and the inadequate secretion of endogenous glucocorticoids is also detectable by studying serum levels of cytokines and steroids during the circadian rhythms.

Interestingly, the circadian rhythm of serum cortisol with respect to amplitude and period is similar in both healthy controls and in RA patients having a mild or moderate disease. On the contrary serum levels of IL-6 are at least ten times higher as well as the circadian rhythm is quite different in healthy controls versus RA patients.

However, despite the raised serum concentrations of IL- 6 in RA active disease, the amplitude of the circadian rhythm of cortisol is not increased as should be expected. This condition confirms an of inadequate cortisol secretion in presence of adrenal stress that in turn is related to persistent active disease [18] (Fig. 2). 
In addition, the degradation of the bioactive cortisol to the biologically inactive cortisone it has been shown increased when studying mixed synovial cell populations originated by the inflamed tissue. This phenomenon has been found due to increased numbers of positive cell for the degrading enzyme $11 \beta$-hydroxysteroid dehydrogenase (type 2) and to a decreased reactivation of cortisone to cortisol [19].

As matter of fact, the clinical improvement observed in RA patients after glucocorticoid therapy appeared in a previous study, to be attributed to a direct dampening of pro-inflammatory factors, as well as to the restoration of the steroid milieu [20].

In conclusion, since cortisol is considered the most potent endogenous anti-inflammatory substance, its pathological downregulation and hyposecretion during the night, justify the appearance of the early morning clinical symptoms in RA patients, while in contrast the perseverant synthesis of melatonin may continous to enhance the night inflammatory reaction [20].

\section{The production and the role of melatonin in rheumatoid arthritis}

Melatonin (nocturnal pineal gland hormone) exerts a variety of actions on the immune system. Like cortisol, melatonin production follows a circadian rhythm, but activates immune cells and enhances inflammatory cytokine and nitric oxide [5, 21].

Previous investigations have shown that in both in healthy subjects and RA patients, melatonin levels increase progressively from $8 \mathrm{pm}$ to the early morning hours and in RA patients they reach peak levels at midnight, which was at least 2 hours earlier than in healthy controls [21].

Consequently, in RA patients, melatonin concentrations reach a plateau that last for $2-3$ hours and that is not observed in controls whereas, after 2 am, melatonin levels decrease similarly both in healthy subjects and in RA patients.

Most detailed investigations, confirmed that the nocturnal rhythm of melatonin occurs also in RA patients, but with an earlier nocturnal peak and a with a longer duration in the early morning $[5,21]$.

Indeed, the production of several cytokines, like IL-1, IL-6, tumour necrosis factor $\alpha$ (TNF $\alpha$ ), IFN- $\gamma$, IL-2, and IL-12 (Th1 cytokines) reach their peak during the night, shortly later the melatonin serum levels are highest and plasma cortisol is lowest.

Of real interest, melatonin was found detectable and in high concentration in synovial fluids from RA patients, and binding sites for melatonin were found present in synovial macrophages [22]. In addition, cultured RA syno- vial macrophages react to melatonin stimulation with an increased production of proinflammatory cytokine [23]). In conclusion, it is not surprising that melatonin administration does not improve RA clinical status, but on the contrary may further enhance the nocturnal inflammatory reaction as previously showed [24].

\section{Low-dose glucocorticoids and chronotherapy in rheumatoid arthritis}

The treatment with exogenous glucocorticoids (i.e. prednisone) is today recommended at low doses in RA since may act like a "replacement therapy" in presence of decreased endogenous cortisol availability, as previously discussed $[25,26]$.

Clearly, important aspects still differentiate between exogenous (i.e. therapeutic) and endogenous (i.e. physiologic) glucocorticoids, especially regarding different mineralocorticoid and glucocorticoid activities [25].

As matter of fact, exogenous synthetic glucocorticoids exert a more selected glucocorticoid/antiinflammatory action (less mineralcorticoid effects), as well as have different plasma kinetics, metabolism, biologic half-life and (nongenomic) high-dose effects compared to cortisol (or hydrocortisone).

However, in any case, the long-term administration of exogenous glucocorticoid may interfere with the HPA axis function and as consequence with the circadian cortisol (endogenous) production.

A recent analysis considering the period 1980-2004 and regarding the long-term glucocorticoid therapy in RA, has shown in the last decades, a progressive reduction of mean initial low-dose from 10.3 up to $3.6 \mathrm{mg} /$ day (prednisone) [27].

The more specific items of the EULAR recommendations for the management of RA relate, when starting disease-modifying antirheumatic drug (DMARD) therapy in early disease, to use a conventional DMARD strategy in combination with (low) doses glucocorticoids [28]. This approach (low dose glucocorticoids) has been approved very recently and suggested also in the new guide lines of the ACR [29].

As matter of fact, in agreement with the conventional definition of DMARD, there is evidence that glucocorticoid therapy in RA, especially long-term low-dose treatment, may in addition slow radiographic progression by at least $50 \%$ when given to patients with early disease.

Since glucocorticoids exert crucial genomic effects on cellular immunity and given the presence of the cellular circadian rhythms, therefore, the prevention of the pathological nocturnal up-regulation of immune cell activity (and related increased production of cytokines), 
may be not optimal with their exogenous administration betwen 6.00 and 8.00 since too late [30].

Since it is well evident that RA symptoms like pain, stiffness and functional disability show maximum level in the early morning hours, it is now also evident that preventing the nocturnal rise of proinflammatory cytokines by night glucocorticoids is more effective than treating established symptoms in the morning [31].

Finally, in RA, several inflammatory pathways involving also the central nervous system (i.e. involved in pain perception), might be better controlled by the correct chronotherapy and have been shown to result on increased sleep quality and also reduction of depressive symptoms [6].

Therefore, in 1964 was published the first reliable clinical study showing the superiority of night versus morning administration of glucocorticoids in RA [32]. In detail, fifty-six RA patients were included in a double-blind trial and none of patients had at any time taken a dose of glucocorticoids superior to $5 \mathrm{mg}$ prednisolone. For each RA patient the 4-week trial was divided into 2 -week periods and RA patients received one tablet at $10 \mathrm{pm}$ (5 mg prednisolone) and a second tablet-placebo, in the morning. As result the $5 \mathrm{mg}$ prednisolone administered at night was found to reduce or eliminate morning stiffness in a superior manner in the majority of RA patients. The effect was fast and, according to the study design, it was confirmed by altering in the patients the time of administration from night to morning and vice versa in random style and under double-blind method [32].

Twenty years later (1984), a further study involved 41 RA patients treated with low-dose prednisolone (mean $5.8 \mathrm{mg}$ ) and again included in a double-blind cross-over study with the intention to establish the effect of timing (morning or night) of prednisolone dosage on morning symptoms (especially stiffness) [33].

The RA patients received prednisolone $(70 \%=$ or $<5 \mathrm{mg} /$ day) on the late evening (10-11 pm) and on rising (6-7 am) and once again prednisolone given at night induced a significantly shorter duration of morning stiffness ( $p=0.0001$ ) versus the equivalent dose given in the morning. In addition, a significant patient preference ( $p<0.05)$ for the night therapy using the non-parametric statistical analysis (sign test).

In 1997, 26 RA patients glucocorticoid-naive, were in random manner tested into two equal groups and treated with to low doses of prednisolone at either $2.00 \mathrm{am}$ or 7.30 am [34].

Again low doses of prednisolone (5 or $7.5 \mathrm{mg}$ daily) at 2.00 am after only 5 days showed superior efficacy on the duration of morning stiffness $(p<0.001)$, Lansbury index $(p<0.001)$, Ritchie index $(p<0.001)$, joint pain $(p<0.001)$ and morning serum concentrations of IL-6 ( $p<0.01)$.
Interestingly, the other study group, showed minor effects, even if significant, on morning stiffness ( $p<$ $0.05)$ and circulating concentrations of IL-6 ( $p<0.05)$.

More recently, using a most advanced approach for the low-dose prednisone chronotherapy in RA patients included the modified-release prednisone, consisting in a timing drug release with administration at $10 \mathrm{pm}$ and release around 2-3 am [35].

Large-scale trials in RA patients documented that modified-release prednisone has superior efficacy for long-term low-dose glucocorticoid treatment, showing a significant reduction in morning joint stiffness/fatigue and a similar safety profile but without any additional suppression of the HPA axis [36, 37].

All the investigations confirmed that the specific timing of exogenous prednisone availability, linked to the interaction between IL-6 and the HPA axis, may correct in RA a postulated deficiency regarding the HPA control [38].

\section{Management results from the glucocorticoid chronotherapy in rheumatoid arthritis}

Recent single-center crossover studies were conducted in RA patients in order to compare the pharmacokinetics of a single $5 \mathrm{mg}$ oral dose of modified-release prednisone and conventional prednisone [39].

No significant differences in pharmacokinetic parameters of both formulations apart from the programmed delay in release of prednisone from the night-time-release formulation were detected.

The effects on the HPA axis of long-term low-dose chronotherapy with modified-release prednisone have been carefully investigated in RA patients [40]. The increase of cortisol plasma concentrations after injection of corticotropin-releasing hormone (CRH) was 5.5 (SD 4.37) $\mu \mathrm{g} / \mathrm{dl}$ on regular-morning prednisone after 3 months and 5.3 (4.07) $\mathrm{kg} / \mathrm{dl}$ on modified-release prednisone at 12 months.

In addition, no worsening of adrenal impairment was found on treatment with night-time-release prednisone in RA patients with low responsiveness to $\mathrm{CRH}$ testing before the treatment with such approach.

Interestingly, an observational study assessed functional ability in RA patients treated with modified-release prednisone under conditions of normal clinical practice [41] and the dose of prednisone was significantly reduced after 9 months of treatment (from 5.0 to 4.4 $\mathrm{mg} /$ day, $p<0.001$ ) with modified-release prednisone, as well as fewer RA patients required biological DMARD treatments. 
To assess if modified-release prednisone administered at bedtime is confirmed more efficient than prednisone administered in the morning, a total number of 950 RA outpatients treated with different glucocorticoids and DMARDs (10.5\% leflunomide, $83.7 \%$ methotrexate, $15.8 \%$ biologics) were switched from morning prednisone or 6-methyl (6M)-prednisolone to low-dose modified-release prednisone and were followed for 4 months [42].

In particular, 513 RA patients were switched to modified-release prednisone from morning prednisone (9.4 $\pm 5.4 \mathrm{mg}$ ) and 437 from $6 \mathrm{M}$-prednisolone $(6.7 \pm 3.7 \mathrm{mg})$. After 4-months $(904,96.8 \%)$ of modified-release prednisone treatment following the switch from morning prednisone or $6 \mathrm{M}$-prednisolone, patient and physician global assessment, morning stiffness duration and pain intensity improved significantly $(p<0.001)$ when compared to basal time. Finally, mean daily modified-release prednisone doses also decreased from $8.2 \mathrm{mg}$ to $6.7 \mathrm{mg}$ between baseline and the check at 4 months.

It was observed that glucocorticoid-naive RA patients seem the best responders to the night-time-release prednisone, however, whereas switching from other glucocorticoids to low dose modified-release prednisone it seems to improve significantly outcomes especially after 4 months, as reported in the open, un-blinded study [42].

To confirm these studies, in a very recent investigation assessing the efficacy and safety of night-time-release prednisone versus morning prednisone in newly diagnosed polymyalgia rheumatica (PMR) patients, there was a clear consistent trend for a significantly stronger effect of modified-release prednisone across most secondary efficacy endpoints at week 4 , with a evident treatment differences observed already at week 1 [43].

Of note, night-time-release prednisone reduced IL-6 concentrations more significantly in morning-release prednisone treated patients $(p<0.01)$.

Cost-effectiveness of modified-release formulations compared with immediate-release prednisone has been also evaluated [44]. Health benefits were evaluated as quality-adjusted life years (QALYs) and the final output was the incremental cost-effectiveness ratio (ICER).

As expected, mean treatment costs per patient were found higher for night-time-release than for immediate-release prednisone. In particular, the analysis showed that mean treatment costs per patient per year is higher for modified release-prednisone ( $£$ 649.70) than for regular prednisone ( $£ 46.54)$.

On the other hands, the model generated an incremental QALY of 0.044 in favor of modified-release prednisone which resulted in an ICER of $£ 13,577$.
Probabilistic sensitivity analysis showed that modified release-prednisone induces an $84 \%$ probability of being cost-effective if there is a willingness-to-pay threshold of $£ 30,000$ per QALY.

Another study on cost-effectiveness, analyzed the effects of a 12-week treatment with modified-release prednisone versus placebo on the costs of drug treatment of RA [45]. The study concluded that $11-13 \%$ more patients on modified-release prednisone than on placebo dropped below reimbursement thresholds for the UK, Belgium and Netherlands and assuming 1 year of biologics cost $€ 15,000$ and MR-pred costs $€ 1$ /day, almost $€ 396$ are saved in each RA patient since delaying biologic treatment by 12 weeks.

\section{Other approaches to chronotherapy in rheumatoid arthritis}

Different cells involved in the inflammatory process (i.e. monocytes that loose their normal circadian rhythms) are particularly activated during the night, therefore, other therapeutical approaches used in RA, for example with non- steroidal anti-inflammatory drugs (NSAIDs) and DMARDs should follow the same concepts of glucocorticoid chronotherapy [46].

Of note, the circadian activation of the cells involved in the RA immune/inflammatory process represent the preferential target for conventional and biologic DMARDs, and the administration of anti-proliferative drugs (i.e. methotrexate, leflunomide, cyclophosphamide etc.) should be adapted to those rhythms.

This possible approach was tested in an in vivo investigation using an animal model of arthritis, and confirmed that the optimal dosing time for the methotrexate anti-inflammatory action and decrease of TNF- $\alpha$, should be associated when adapting to the 24-h cycling of TNF- $\alpha$, itself [47].

A further clinical study has suggested that bedtime methotrexate chronotherapy (3 times a week once a day the evening) can better improve RA symptoms compared to the current standard dosing methods [48].

Interesting, the migration/activation of neutrophilic polymorphonuclear in the inflammatory areas, follows night rhythms and starting early in the 1980s, various controlled-release NSAIDs have been explored for administration-time differences in their symptomatic effects [49].

An early double-blind, crossover study design that included multiple (4 to 6 times per day) pain, stiffness, and hand strength self-assessments in RA patients, showed that a twice-daily flurbiprofen schedule (i.e. $200 \mathrm{mg}$ in the morning and $200 \mathrm{mg}$ at midday) was less effective in modulating morning RA signs and symptoms than 
ones that included bed time administration $(200 \mathrm{mg}$ in the morning and $200 \mathrm{mg}$ at bedtime or $200 \mathrm{mg}$ at midday and $200 \mathrm{mg}$ at bedtime) [50].

A further study, found that evening once-daily scheduling of $75 \mathrm{mg}$ (indomethacin formulation $25 \mathrm{mg}$ immediate-release combined with $50 \mathrm{mg}$ controlled-release) resulted in a more efficient control of morning OA symptoms compared to once-daily morning or once-daily midday [51].

Interestingly, a $\mathrm{pH}$-responsive dual pulse multiparticulate dosage compund containing ketoprofen, was tested in RA and was found able to control circadian symptoms during midnight and early morning [52].

In addition, with the main intent of delivering maximum concentration of indomethacin in the night, an oral compression coated tablet was developed with a lag time of 6 hours, before immediate release [53].

Further results should be obtainable in chronopharmacological treatment of morning RA symptoms with a new synthesized eudragit-coated aceclofenac-loaded pectin microspheres or even with with a $\mathrm{pH}$-triggered delayed-release colon-specific aceclofenac microspheres [54, 55].

Finally, a formulation of mini-tablets-filled-pulsincap delivering lornoxicam for chronotherapy of RA was synthesizedfor the night administration [56]. The optimized pulsincap formulation releases lornoxicam after a lag time of 5 hrs with maximum amount of the drug available in the early morning.

In conclusion, the recent research on biological rhythms in inflammation and the synthesis of "circadian"drugs, is leading to a better understanding of the mechanisms of inflammation and to an optimal use of the drugs in RA [57, 58].

\section{Conclusions}

The stiffness and functional joint disability characterizing the early morning hours in RA patients, is related to the night activation of the immune/inflammatory response.

The prevention/treatment of the immune cell activity (and related flare of cytokine synthesis and other mediators) has been shown more effective when the availability of the exogenous glucocorticoids is obtained in the middle of the night. The very positive results obtained in RA patients treated with modified-release prednisone for the low dose chronotherapy, and following the chronobiology of the disease, seem applicable even for other agents such as conventional DMARDs and NSAIDs.

\section{Acknowledgements}

We that Dr. Sara DeGregorio for her help in graphic management.
The authors declare no conflict of interest.

\section{References}

1. Cutolo M, Villaggio B, Otsa K, et al. Altered circadian rhythms in rheumatoid arthritis patients play a role in the disease's symptoms. Autoimmun Rev 2005; 4: 497-502.

2. Straub RH, Cutolo M. Circadian rhythms in rheumatoid arthritis: implications for pathophysiology and therapeutic management. Arthritis Rheum 2007; 56: 399-408.

3. Spies CM, Cutolo M, Straub RH, et al. More night than day circadian rhythms in polymyalgia rheumatica and ankylosing spondylitis. J Rheumatol 2010; 37: 894-899.

4. Straub RH, Bijlsma JW, Masi A, et al. Role of neuroendocrine and neuroimmune mechanisms in chronic inflammatory rheumatic diseases - the 10-year update. Semin Arthritis Rheum 2013; 43: 392-404.

5. Cutolo M, Seriolo B, Craviotto C, et al. Circadian rhythms in RA. Ann Rheum Dis 2003; 62: 593-596.

6. Buttgereit F, Smolen JS, Coogan AN, et al. Clocking in: chronobiology in rheumatoid arthritis. Nat Rev Rheumatol 2015; 11: 349-356.

7. Cutolo M. Rheumatoid arthritis: circadian and circannual rhythms in RA. Nat Rev Rheumatol 2011; 7: 500-502.

8. Bijlsma JW, Cutolo M, Masi AT, et al. The neuroendocrine immune basis of rheumatic diseases. Immunol Today 1999; 20: 298-301.

9. Gibbs JE, Ray DW. The role of the circadian clock in rheumatoid arthritis. Arthritis Res Ther 2013; 15: 205.

10. Albrecht U. Timing to perfection: the biology of central and peripheral circadian clocks. Neuron 2012, 74: 246-260.

11. Coogan AN, Wyse CA. Neuroimmunology of the circadian clock. Brain Res 2008; 1232: 104-112.

12. Wang XS, Armstrong ME, Cairns BJ, et al. Shift work and chronic disease: the epidemiological evidence. Occup Med (Lond) 2011; 61: 78-89.

13. Puttonen S, Oksanen T, Vahtera J, et al. Is shift work a risk factor for rheumatoid arthritis? The Finnish Public Sector study. Ann Rheum Dis 2010; 69: 779-780.

14. Sulli A, Maestroni GJ, Villaggio B, et al. Melatonin serum levels in rheumatoid arthritis. Ann NY Acad Sci 2002; 966: 276-283.

15. Cutolo $M$, Straub RH, Buttgereit F. Circadian rhythms of nocturnal hormones in rheumatoid arthritis: translation from bench to bedside. Ann Rheum Dis 2008; 67: 905-908.

16. Kanczkowski W, Sue M, Zacharowski K, et al. The role of adrenal gland microenvironment in the HPA axis function and dysfunction during sepsis. Mol Cell Endocrinol 2015; 408: 241-248.

17. Annetta M, Maviglia R, Proietti R, et al. Use of corticosteroids in critically ill septic patients: a review of mechanisms of adrenal insufficiency in sepsis and treatment. Curr Drug Targets 2009; 10: 887-894.

18. Crofford LJ, Kalogeras KT, Mastorakos G, et al. Circadian relationships between interleukin (IL)- 6 and hypothalamic-pituitary-adrenal axis hormones: failure of IL-6 to cause sustained hypercortisolism in patients with early untreated rheumatoid arthritis. J Clin Endocrinol Metab 1997; 82: 1279-1283. 
19. Schmidt M, Weidler C, Naumann H, et al. Reduced capacity for the reactivation of glucocorticoids in rheumatoid arthritis synovial cells: Possible role of the sympathetic nervous system? Arthritis Rheum 2005; 52: 1711-1120.

20. Cutolo M, Foppiani L, Minuto F. Hypothalamic-pituitary-adrenal axis impairment in the pathogenesis of rheumatoid arthritis and polymyalgia rheumatica. J Endocrinol Invest 2002; 25: $19-23$

21. Sulli A, Maestroni GJM, Villaggio B, et al. Melatonin serum levels in rheumatoid arthritis. Ann N Y Acad Sci 2002; 966 276-278.

22. Maestroni GJM, Sulli A, Villaggio B, et al. Melatonin in rheumatoid arthritis: synovial macrophages show melatonin receptors. Ann N Y Acad Sci 2002; 966: 271-275.

23. Cutolo $M$, Villaggio $B$, Candido $F$, et al. Melatonin influences interleukin-12 and nitric oxide production by primary cultures of rheumatoid synovial macrophages and THP-1 cells. Ann N Y Acad Sci 1999; 876: 246-254.

24. Maestroni GJ, Otsa K, Cutolo M. Melatonin treatment does not improve rheumatoid arthritis. Br J Clin Pharmacol 2008; 65: 797-798.

25. Buttgereit F, Burmester GR, Straub RH, et al. Exogenous and endogenous glucocorticoids in rheumatic diseases. Arthritis Rheum 2011; 63: 1-9.

26. Gorter SL, Bijlsma JW, Cutolo M, et al. Current evidence for the management of rheumatoid arthritis with glucocorticoids: a systematic literature review informing the EULAR recommendations for the management of rheumatoid arthritis. Ann Rheum Dis 2010; 69: 1010-1014.

27. Pincus T, Sokka T, Cutolo $M$. The past versus the present, 1980-2004: reduction of mean initial low-dose, long-term glucocorticoid therapy in rheumatoid arthritis from 10.3 to $3.6 \mathrm{mg} / \mathrm{day}$, concomitant with early methotrexate, with long term effectiveness and safety of less than $5 \mathrm{mg} /$ day. Neuroimmunomodulation 2015; 22: 89-103.

28. Smolen JS, Landewé R, Bijlsma J, et al. EULAR recommendations for the management of rheumatoid arthritis with synthetic and biological disease-modifying antirheumatic drugs: 2016 update. Ann Rheum Dis 2017; 76: 960-977.

29. Singh JA, Saag KG, Bridges SL Jr, et al. 2015 American College of Rheumatology Guideline for the Treatment of Rheumatoid Arthritis. Arthritis Rheum 2016; 68: 1-26.

30. Spies CM, Cutolo M, Straub RH et al. Prednisone chronotherapy. Clin Exp Rheumatol 2011; 29 (5 Suppl 68): S42-536.

31. Cutolo M, Otsa K, Aakre O, et al. Nocturnal hormones and clinical rhythms in rheumatoid arthritis. Ann N Y Acad Sci 2005 1051: 372-381.

32. De Andrade JR, McCormick JN, Hill AG. Small doses of prednisolone in the management of rheumatoid arthritis. Ann Rheum Dis 1964; 23: 158-162.

33. De Silva M, Binder A, Hazleman BL. The timing of prednisolone dosage and its effect on morning stiffness in rheumatoid arthritis. Ann Rheum Dis 1984; 43: 790-793.

34. Arvidson NG, Gudbjörnsson B, Larsson A, et al. The timing of glucocorticoid administration in rheumatoid arthritis. Ann Rheum Dis 1997; 56: 27-31.

35. Buttgereit F, Doering G, Shaeffler A, et al. Efficacy of modified-release versus standard prednisone to reduce duration of morning stiffness of the joints in rheumatoid arthritis (CAPRA-1): a double-blind, randomised controlled trial. Lancet 2008; 371: 205-214.

36. Buttgereit F, Doering G, Schaeffler A, et al. Targeting pathophysiological rhythms: prednisone chronotherapy shows sustained efficacy in rheumatoid arthritis. Ann Rheum Dis 2010; 69: $1275-1280$

37. Alten R, Grahn A, Holt RJ, et al. Delayed-release prednisone improves fatigue and health-related quality of life: findings from the CAPRA-2 double-blind randomised study in rheumatoid arthritis. RMD Open 2015; 1: e000134.

38. Cutolo M, Sulli A, Pincus T. Circadian use of glucocorticoids in rheumatoid arthritis. Neuroimmunomodulation 2015; 22 : 33-39.

39. Derendorf H, Ruebsamen K, Clarke L, et al. Pharmacokinetics of modified-releaseprednisone tablets in healthy subjects and patients with rheumatoid arthritis. J Clin Pharmacol 2013; 53 : 326-333.

40. Alten R, Döring G, Cutolo M, et al. Hypothalamus-pituitary-adrenal axis function in patients with rheumatoid arthritis treated with nighttime-release prednisone. J Rheumatol 2010; 37: 2025-2031.

41. Pfeiffer BM, Krenzer S, Dockhorn R, et al. Impact of modified-release prednisone on functional ability in patients with rheumatoid arthritis. Rheumatol Int 2013; 33: 1447-1454.

42. Cutolo M, laccarino L, Doria A, et al. Efficacy of the switch to modified-release prednisone in rheumatoid arthritis patients treated with standard glucocorticoids. Clin Exp Rheumatol 2013; 31: 498-505.

43. Cutolo M, Hopp M, Liebscher S, et al. Efficacy and safety of modified release prednisone (Lodotra ${ }^{\circledR}$ ) in patients with polymyalgia rheumatica: results of a multicenter, randomized, active-controlled phase 3 Study. Arthritis Rheum 2015; 67: S10-3145.

44. Dunlop W, Iqbal I, Khan I, et al. Cost-effectiveness of modified-release prednisone in the treatment of moderate to severe rheumatoid arthritis with morning stiffness based on directly elicited public preference values. Clinicoecon Outcomes Res 2013; 5: 555-564.

45. Boers $M$, Buttgereit F. A simple model that suggests possible cost savings when modified-releaseprednisone $5 \mathrm{mg}$ /day is added to current treatment in patients with active rheumatoid arthritis. Rheumatology (Oxford) 2013; 52: 1435-1437.

46. Spies CM, Hoff P, Mazuch J. Circadian rhythms of cellular immunity in rheumatoid arthritis: a hypothesis-generating study. Clin Exp Rheumatol 2015; 33: 34-43.

47. To $\mathrm{H}$, Irie S, Tomonari $\mathrm{M}$, et al. Therapeutic index of methotrexate depends on circadian cycling of tumour necrosis factor-alpha in collagen-induced arthritic rats and mice. J Pharm Pharmacol 2009; 61: 1333-1338.

48. To $\mathrm{H}$, Yoshimatsu $\mathrm{H}$, Tomonari $\mathrm{M}$, et al. Methotrexate chronotherapy is effective against rheumatoid arthritis. Chronobiol Int 2011; 28: 267-274.

49. Bureau JP, Labrecque G. Biological rhythm, inflammation and non-steroidal anti-inflammatory agents. Pathol Biol (Paris) 1996; 44: 610-617.

50. Kowanko IC, Pownall R, Knapp MS, et al. Circadian variations in the signs and symptoms of rheumatoid arthritis and in the 
therapeutic effectiveness of flurbiprofen at different times of day. Br J Clin Pharmacol 1981; 11: 477-484.

51. Levi F, Le Louarn C, Reinberg A. Timing optimizes sustained-release indomethacin treatment of osteoarthritis. Clin Pharmacol Ther 1985; 37: 77-84.

52. Lotlikar V, Kedar U, Shidhaye S, et al. pH-responsive dual pulse multiparticulate dosage form for treatment of rheumatoid arthritis. Drug Dev Ind Pharm 2010; 36: 1295-1302.

53. Sunil SA, Srikanth MV, Rao NS, et al. Chronotherapeutic drug delivery from indomethacin compression coated tablets for early morning pain associated rheumatoid arthritis. Curr Drug Deliv 2013; 10: 109-121.

54. Ramasamy T, Ruttala HB, Shanmugam S, et al. Eudragit-coated aceclofenac-loaded pectin microspheres in chronopharmacological treatment of rheumatoid arthritis. Drug Deliv 2013; 20: 65-77.

55. Sanka K, Pragada RR, Veerareddy PR. A pH-triggered delayed-release chronotherapeutic drug delivery system of aceclofenac for effective management of early morning symptoms of rheumatoid arthritis. J Microencapsul 2015; 32: 794.

56. Hadi MA, Rao NG, Rao AS. Formulation and evaluation of minitablets-filled-pulsincap delivery of lornoxicam in the chronotherapeutic treatment of rheumatoid arthritis. Pak J Pharm Sci 2015; 28: 185-193.

57. Masi AT, Bijlsma JW, Chikanza IC, et al. Neuroendocrine, immunologic, and microvascular systems interactions in rheumatoid arthritis: physiopathogenetic and therapeutic perspectives. Semin Arthritis Rheum 1999; 29: 65-81.

58. Straub RH, Glück T, Cutolo $M$, et al. The adrenal steroid status in relation to inflammatory cytokines (interleukin- 6 and tumour necrosis factor) in polymyalgia rheumatica. Rheumatology (Oxford) 2000; 39 (6): 624-631. 